\title{
On a Time-Fractional Integrodifferential Equation via Three-Point Boundary Value Conditions
}

\author{
Dumitru Baleanu, ${ }^{1,2}$ Shahram Rezapour, ${ }^{3}$ Sina Etemad, ${ }^{3}$ and Ahmed Alsaedi ${ }^{4}$ \\ ${ }^{1}$ Department of Mathematics, Cankaya University, Ogretmenler Caddesi 14, Balgat, 06530 Ankara, Turkey \\ ${ }^{2}$ Institute of Space Sciences, Magurele, Bucharest, Romania \\ ${ }^{3}$ Department of Mathematics, Azarbaijan Shahid Madani University, Azarshahr, Tabriz, Iran \\ ${ }^{4}$ Department of Mathematics, Faculty of Science, King Abdulaziz University, Jeddah 21589, Saudi Arabia
}

Correspondence should be addressed to Dumitru Baleanu; dumitru.baleanu@gmail.com

Received 15 July 2014; Revised 15 October 2014; Accepted 20 October 2014

Academic Editor: Guido Maione

Copyright (C) 2015 Dumitru Baleanu et al. This is an open access article distributed under the Creative Commons Attribution License, which permits unrestricted use, distribution, and reproduction in any medium, provided the original work is properly cited.

The existence and the uniqueness theorems play a crucial role prior to finding the numerical solutions of the fractional differential equations describing the models corresponding to the real world applications. In this paper, we study the existence of solutions for a time-fractional integrodifferential equation via three-point boundary value conditions.

\section{Introduction}

There is no doubt that the fractional calculus has various important applications in many fields as mathematics (see the following monographs $[1,2]$ and the references therein), physics [3], and economics [4], as well as in many other branches of science and engineering $[3,5,6]$. Recently the numerical methods were applied intensively to solve complicated fractional differential equations (FDE) which describe the real world applications. As an example, we mention that several physical processes exhibit fractional order behavior varying with time and/or space [7]. Thus, a class of phenomena can be obtained by analyzing this most general model. A fundamental step is to prove the existence and uniqueness of the proposed fractional nonlinear differential equations for these models. There are many works on fractional differential equations and inclusions. The existence of solutions for integrodifferential equations of fractional order with nonlocal three-point fractional boundary conditions was developed in [8] while the importance of antiperiodic type integral boundary conditions was discussed in [9]. The existence of solutions for fractional differential inclusions in the presence of the separated boundary conditions in Banach space was developed in [10]. We recall that the existence and multiplicity of positive solutions for singular fractional boundary value problems were analyzed in [11]. The existence and multiplicity of positive solutions for singular fractional boundary value problems were the topic debated in [12]. Making use of the fixed point results on cones, the existence and uniqueness of positive solutions for some nonlinear fractional differential equations were developed in [13]. Further results on positive solutions of a boundary value problem for nonlinear fractional differential equations were reported in [14] and new results on the existence results on nonlinear fractional differential equations were reported in [15]. For other related results we suggest for the readers $[16,17]$ as well as the references therein. We recall that the existence of solutions for some fractional partial differential equations was investigated in $[18,19]$ and the references therein.

Let $n$ be a natural number, $n-1<\alpha<n, a, b \in$ $\mathbb{R}$, and $u \in C([a, b] \times[a, b], \mathbb{R})$. The Riemann-Liouville time-fractional order integral of the function $u$ is defined by $I_{* t}^{\alpha} u(x, t)=(1 / \Gamma(\alpha)) \int_{0}^{t}\left(u(x, \tau) /(t-\tau)^{1-\alpha}\right) d \tau$ for all $x, t \in$ $[a, b]$ whenever the integral exists (more details regarding the basic definitions of the fractional calculus can be seen in [1]). Also, the Caputo derivative of time-fractional of order 
$\alpha$ for the function $u$ is defined by ${ }^{c} D_{* t}^{\alpha} u(x, t)=(1 / \Gamma(n-$ $\alpha)) \int_{0}^{t}(t-\tau)^{n-\alpha-1}\left(\partial^{n} u(x, \tau) / \partial \tau^{n}\right) d \tau$ (see for more details [1]). It has been proved that the general solution of the timefractional differential equation ${ }^{c} D_{* t}^{\alpha} u(x, t)=0$ is given by $u(x, t)=c_{0}+c_{1} t+c_{2} t^{2}+\cdots+c_{n-1} t^{n-1}$, where $c_{0}, \ldots, c_{n-1}$ are real constants and $n=[\alpha]+1$ (see [20]). Also, for each $T>0$ and $u \in C([0, T] \times[0, T])$ we have $I_{* t}^{\alpha c} D_{* t}^{\alpha} u(x, t)=$ $u(x, t)+c_{0}+c_{1} t+c_{2} t^{2}+\cdots+c_{n-1} t^{n-1}$, where $c_{0}, \ldots, c_{n-1}$ are real constants and $n=[\alpha]+1([20])$. Let $M$ be a bounded set in a metric space $(X, d)$. Then the Kuratowski measure of noncompactness is defined by (see [21])

$\alpha(M)=\inf \{\epsilon: M$ covered by finitely many sets in which the diameter of each set is less than or equal to $\epsilon\}$.

Let $\Phi: D(\Phi) \subseteq X \rightarrow X$ be a bounded and continuous operator on a Banach space $X$. Then $\Phi$ is called a condensing map whenever $\alpha(\Phi(B))<\alpha(B)$ for all bounded sets $B \subset D(\Phi)$, where $\alpha$ denotes the Kuratowski measure of noncompactness (see [21]).

In this paper, we study the existence of solutions for a time-fractional integrodifferential equation via three-point boundary value conditions under certain conditions. We mention that the investigated equation generalized a huge class of classical ordinary differential equations which can be found in applications in engineering and physics. The boundary conditions used in this paper are as general as possible and for particular cases of the parameters we recover several cases of fractional nonlinear differential equations. In this way, we use the next Lemma and Sadovskii's fixed point theorem for condensing maps (see [22]).

Lemma 1 (see [23]). Let $X$ be a Banach space, $D \subseteq X, k \in$ $[0,1), K: D \rightarrow X$ a $k$-contraction, and $C: D \rightarrow X a$ compact operator. Then $K+C$ is a $k$-set contraction and so a condensing map.

Theorem 2 (see [22]). Let $B$ be a convex, bounded, and closed subset of a Banach space $X$ and $\Phi: B \rightarrow B$ a condensing map. Then $\Phi$ has a fixed point.

\section{Main Results}

Suppose that $J=[0,1] \times[0,1], 1 \leq q<2$, and $X=\{u$ : $\left.u,\left(\partial^{q} / \partial t^{q}\right) u \in C(J, \mathbb{R})\right\}$ endowed via the norm

$$
\|u\|=\sup _{(x, t) \in J}|u(x, t)|+\sup _{(x, t) \in J}\left|\frac{\partial^{q}}{\partial t^{q}} u(x, t)\right|,
$$

where $\left(\partial^{q} / \partial t^{q}\right) u(x, t)$ denotes the standard Caputo timefractional derivative. Let $2 \leq \alpha<3,0<\beta<1,1 \leq \delta<2$, $0 \leq \eta \leq 1$, and $\lambda, \mu \in \mathbb{R}$, and $f: J \times X^{2} \rightarrow X$ and $g: J \times X \rightarrow X$ are continuous functions. We investigate the nonlinear time-fractional integrodifferential equation as follows:

$$
\begin{aligned}
-\frac{\partial^{\alpha}}{\partial t^{\alpha}} u(x, t)= & \lambda f\left(x, t, u(x, t), \frac{\partial^{q}}{\partial t^{q}} u(x, t)\right) \\
& +\mu I_{* t}^{\beta} g(x, t, u(x, t))
\end{aligned}
$$

via the three-point boundary value conditions $\left(\partial^{\delta+1} / \partial t^{\delta+1}\right)$ $u(x, 0)=\left(\partial^{\delta+1} / \partial t^{\delta+1}\right) u(x, 1),\left(\partial^{\delta} / \partial t^{\delta}\right) u(x, 1)-\left(\partial^{\delta} / \partial t^{\delta}\right)$ $u(x, \eta)=0$, and $u(x, 0)=0$. In this way, we give first next result.

Lemma 3. An element $u_{0}$ in $X$ is a solution for the problem (*) via the three-point boundary value conditions if and only if $u_{0}$ is a solution for the time-fractional integral equation

$$
\begin{gathered}
u(x, t)=\int_{0}^{1} G_{x}(t, \tau)\left[\lambda f\left(x, \tau, u(x, \tau), \frac{\partial^{q}}{\partial \tau^{q}} u(x, \tau)\right)\right. \\
\left.+\mu I_{* t}^{\beta} g(x, \tau, u(x, \tau))\right] d \tau,
\end{gathered}
$$

where

$$
\begin{aligned}
& G_{x}(t, \tau) \\
& =-\frac{(t-\tau)^{\alpha-1}}{\Gamma(\alpha)} \\
& +\left(\left[2 t \Gamma(2-\delta)\left(1-\eta^{2-\delta}\right)\right.\right. \\
& \left.\left.\quad+t^{2}\left(\eta^{1-\delta}-1\right) \Gamma(3-\delta)\right](1-\tau)^{\alpha-\delta-2}\right) \\
& \times\left(2 \Gamma(\alpha-\delta-1)\left[\eta^{1-\delta}(2-\delta)+\eta^{2-\delta}(\delta-1)-1\right]\right)^{-1} \\
& +\frac{\left[t^{2}(1-\delta) \Gamma(3-\delta)-2 t \Gamma(3-\delta)\right](1-\tau)^{\alpha-\delta-1}}{2 \Gamma(\alpha-\delta)\left[\eta^{1-\delta}(2-\delta)+\eta^{2-\delta}(\delta-1)-1\right]} \\
& +\frac{\left[2 t \Gamma(3-\delta)-t^{2}(1-\delta) \Gamma(3-\delta)\right](\eta-\tau)^{\alpha-\delta-1}}{\Gamma(\alpha-\delta) 2\left[\eta^{1-\delta}(2-\delta)+\eta^{2-\delta}(\delta-1)-1\right]}
\end{aligned}
$$

whenever $0 \leq \tau \leq \eta \leq t \leq 1$,

$$
\begin{aligned}
& G_{x}(t, \tau) \\
& =-\frac{(t-\tau)^{\alpha-1}}{\Gamma(\alpha)} \\
& \quad+\left(\left[2 t \Gamma(2-\delta)\left(1-\eta^{2-\delta}\right)\right.\right. \\
& \left.\left.\quad+t^{2}\left(\eta^{1-\delta}-1\right) \Gamma(3-\delta)\right](1-\tau)^{\alpha-\delta-2}\right) \\
& \quad \times\left(2 \Gamma(\alpha-\delta-1)\left[\eta^{1-\delta}(2-\delta)+\eta^{2-\delta}(\delta-1)-1\right]\right)^{-1} \\
& +\frac{\left[t^{2}(1-\delta) \Gamma(3-\delta)-2 t \Gamma(3-\delta)\right](1-\tau)^{\alpha-\delta-1}}{2 \Gamma(\alpha-\delta)\left[\eta^{1-\delta}(2-\delta)+\eta^{2-\delta}(\delta-1)-1\right]}
\end{aligned}
$$


whenever $0 \leq \eta \leq \tau \leq t \leq 1$,

$$
\begin{aligned}
G_{x}(t, \tau) & \\
= & \left(\left[2 t \Gamma(2-\delta)\left(1-\eta^{2-\delta}\right)\right.\right. \\
& \left.\left.+t^{2}\left(\eta^{1-\delta}-1\right) \Gamma(3-\delta)\right](1-\tau)^{\alpha-\delta-2}\right) \\
& \times\left(2 \Gamma(\alpha-\delta-1)\left[\eta^{1-\delta}(2-\delta)+\eta^{2-\delta}(\delta-1)-1\right]\right)^{-1} \\
& +\frac{\left[t^{2}(1-\delta) \Gamma(3-\delta)-2 t \Gamma(3-\delta)\right](1-\tau)^{\alpha-\delta-1}}{2 \Gamma(\alpha-\delta)\left[\eta^{1-\delta}(2-\delta)+\eta^{2-\delta}(\delta-1)-1\right]},
\end{aligned}
$$

whenever $0 \leq \eta \leq t \leq \tau \leq 1$,

$$
\begin{aligned}
G_{x}(t, \tau) & \\
= & \left(\left[2 t \Gamma(2-\delta)\left(1-\eta^{2-\delta}\right)\right.\right. \\
& \left.\left.+t^{2}\left(\eta^{1-\delta}-1\right) \Gamma(3-\delta)\right](1-\tau)^{\alpha-\delta-2}\right) \\
& \times\left(2 \Gamma(\alpha-\delta-1)\left[\eta^{1-\delta}(2-\delta)+\eta^{2-\delta}(\delta-1)-1\right]\right)^{-1} \\
& +\frac{\left[t^{2}(1-\delta) \Gamma(3-\delta)-2 t \Gamma(3-\delta)\right](1-\tau)^{\alpha-\delta-1}}{2 \Gamma(\alpha-\delta)\left[\eta^{1-\delta}(2-\delta)+\eta^{2-\delta}(\delta-1)-1\right]},
\end{aligned}
$$

whenever $0 \leq t \leq \eta \leq \tau \leq 1$,

$$
\begin{aligned}
G_{x}(t, \tau) & \\
= & \left(\left[2 t \Gamma(2-\delta)\left(1-\eta^{2-\delta}\right)\right.\right. \\
& \left.\left.+t^{2}\left(\eta^{1-\delta}-1\right) \Gamma(3-\delta)\right](1-\tau)^{\alpha-\delta-2}\right) \\
& \times\left(2 \Gamma(\alpha-\delta-1)\left[\eta^{1-\delta}(2-\delta)+\eta^{2-\delta}(\delta-1)-1\right]\right)^{-1} \\
& +\frac{\left[t^{2}(1-\delta) \Gamma(3-\delta)-2 t \Gamma(3-\delta)\right](1-\tau)^{\alpha-\delta-1}}{2 \Gamma(\alpha-\delta)\left[\eta^{1-\delta}(2-\delta)+\eta^{2-\delta}(\delta-1)-1\right]} \\
& +\frac{\left[2 t \Gamma(3-\delta)-t^{2}(1-\delta) \Gamma(3-\delta)\right](\eta-\tau)^{\alpha-\delta-1}}{\Gamma(\alpha-\delta) 2\left[\eta^{1-\delta}(2-\delta)+\eta^{2-\delta}(\delta-1)-1\right]},
\end{aligned}
$$

whenever $0 \leq t \leq \tau \leq \eta \leq 1$, and

$$
\begin{aligned}
G_{x}(t, \tau) & \\
=- & \frac{(t-\tau)^{\alpha-1}}{\Gamma(\alpha)} \\
+ & \left(\left[2 t \Gamma(2-\delta)\left(1-\eta^{2-\delta}\right)\right.\right. \\
& \left.\left.\quad+t^{2}\left(\eta^{1-\delta}-1\right) \Gamma(3-\delta)\right](1-\tau)^{\alpha-\delta-2}\right)
\end{aligned}
$$

$$
\begin{aligned}
& \times\left(2 \Gamma(\alpha-\delta-1)\left[\eta^{1-\delta}(2-\delta)+\eta^{2-\delta}(\delta-1)-1\right]\right)^{-1} \\
& +\frac{\left[t^{2}(1-\delta) \Gamma(3-\delta)-2 t \Gamma(3-\delta)\right](1-\tau)^{\alpha-\delta-1}}{2 \Gamma(\alpha-\delta)\left[\eta^{1-\delta}(2-\delta)+\eta^{2-\delta}(\delta-1)-1\right]} \\
& +\frac{\left[2 t \Gamma(3-\delta)-t^{2}(1-\delta) \Gamma(3-\delta)\right](\eta-\tau)^{\alpha-\delta-1}}{\Gamma(\alpha-\delta) 2\left[\eta^{1-\delta}(2-\delta)+\eta^{2-\delta}(\delta-1)-1\right]}
\end{aligned}
$$

whenever $0 \leq \tau \leq t \leq \eta \leq 1$.

Proof. Let $u_{0}$ be a solution for the time-fractional integrodifferential equation $(*)$ via the boundary value conditions. Put $y_{0}(x, t)=\lambda f\left(x, t, u_{0}(x, t),\left(\partial^{q} / \partial t^{q}\right) u_{0}(x, t)\right)+$ $\mu I_{* t}^{\beta} g\left(x, t, u_{0}(x, t)\right)$. Choose $c_{0}, c_{1}, c_{2} \in \mathbb{R}$ such that $u_{0}(x, t)=$ $-\int_{0}^{t}\left((t-\tau)^{\alpha-1} / \Gamma(\alpha)\right) y_{0}(x, \tau) d \tau+c_{0}+c_{1} t+c_{2} t^{2}$. Thus, we get

$$
\begin{aligned}
\frac{\partial^{\delta}}{\partial t^{\delta}} u_{0}(x, t)= & -\int_{0}^{t} \frac{(t-\tau)^{\alpha-\delta-1}}{\Gamma(\alpha-\delta)} y_{0}(x, \tau) d \tau \\
& +c_{1} \frac{t^{1-\delta}}{\Gamma(2-\delta)}+c_{2} \frac{2 t^{2-\delta}}{\Gamma(3-\delta)}
\end{aligned}
$$

and $\left(\partial^{\delta+1} / \partial t^{\delta+1}\right) u_{0}(x, t)=-\int_{0}^{t}\left((t-\tau)^{\alpha-\delta-2} / \Gamma(\alpha-\delta-\right.$ 1)) $y_{0}(x, \tau) d \tau+c_{1}\left(t^{-\delta} / \Gamma(1-\delta)\right)+c_{2}\left(2 t^{1-\delta} / \Gamma(2-\delta)\right)$. Now by using the boundary value conditions, we obtain $c_{0}=0$,

$$
\begin{aligned}
c_{1}= & \frac{\Gamma(2-\delta)\left(1-\eta^{2-\delta}\right)}{\left[\eta^{1-\delta}(2-\delta)+\eta^{2-\delta}(\delta-1)-1\right]} \\
& \times \int_{0}^{1} \frac{(1-\tau)^{\alpha-\delta-2}}{\Gamma(\alpha-\delta-1)} y_{0}(x, \tau) d \tau \\
& -\frac{\Gamma(3-\delta)}{\left[\eta^{1-\delta}(2-\delta)+\eta^{2-\delta}(\delta-1)-1\right]} \\
& \times \int_{0}^{1} \frac{(1-\tau)^{\alpha-\delta-1}}{\Gamma(\alpha-\delta)} y_{0}(x, \tau) d \tau
\end{aligned}
$$$$
+\frac{\Gamma(3-\delta)}{\left[\eta^{1-\delta}(2-\delta)+\eta^{2-\delta}(\delta-1)-1\right]}
$$$$
\times \int_{0}^{\eta} \frac{(\eta-\tau)^{\alpha-\delta-1}}{\Gamma(\alpha-\delta)} y_{0}(x, \tau) d \tau
$$

$$
\begin{aligned}
c_{2}= & \frac{(1-\delta) \Gamma(3-\delta)}{2\left[\eta^{1-\delta}(2-\delta)+\eta^{2-\delta}(\delta-1)-1\right]} \\
& \times \int_{0}^{1} \frac{(1-\tau)^{\alpha-\delta-1}}{\Gamma(\alpha-\delta)} y_{0}(x, \tau) d \tau
\end{aligned}
$$




$$
\begin{aligned}
& -\frac{(1-\delta) \Gamma(3-\delta)}{2\left[\eta^{1-\delta}(2-\delta)+\eta^{2-\delta}(\delta-1)-1\right]} \\
& \times \int_{0}^{\eta} \frac{(\eta-\tau)^{\alpha-\delta-1}}{\Gamma(\alpha-\delta)} y_{0}(x, \tau) d \tau \\
& +\frac{\left(\eta^{1-\delta}-1\right) \Gamma(3-\delta)}{2\left[\eta^{1-\delta}(2-\delta)+\eta^{2-\delta}(\delta-1)-1\right]} \\
& \times \int_{0}^{1} \frac{(1-\tau)^{\alpha-\delta-2}}{\Gamma(\alpha-\delta-1)} y_{0}(x, \tau) d \tau .
\end{aligned}
$$

Hence,

$$
\begin{aligned}
u_{0}(x, t)= & -\int_{0}^{t} \frac{(t-\tau)^{\alpha-1}}{\Gamma(\alpha)} y_{0}(x, \tau) d \tau \\
& +\frac{2 t \Gamma(2-\delta)\left(1-\eta^{2-\delta}\right)+t^{2}\left(\eta^{1-\delta}-1\right) \Gamma(3-\delta)}{2\left[\eta^{1-\delta}(2-\delta)+\eta^{2-\delta}(\delta-1)-1\right]} \\
& \times \int_{0}^{1} \frac{(1-\tau)^{\alpha-\delta-2}}{\Gamma(\alpha-\delta-1)} y_{0}(x, \tau) d \tau \\
& +\frac{t^{2}(1-\delta) \Gamma(3-\delta)-2 t \Gamma(3-\delta)}{2\left[\eta^{1-\delta}(2-\delta)+\eta^{2-\delta}(\delta-1)-1\right]} \\
& \times \int_{0}^{1} \frac{(1-\tau)^{\alpha-\delta-1}}{\Gamma(\alpha-\delta)} y_{0}(x, \tau) d \tau \\
& +\frac{2 t \Gamma(3-\delta)-t^{2}(1-\delta) \Gamma(3-\delta)}{2\left[\eta^{1-\delta}(2-\delta)+\eta^{2-\delta}(\delta-1)-1\right]} \\
& \times \int_{0}^{\eta} \frac{(\eta-\tau)^{\alpha-\delta-1}}{\Gamma(\alpha-\delta)} y_{0}(x, \tau) d \tau \\
= & \int_{0}^{1} G_{x}(t, \tau) y_{0}(x, \tau) d \tau .
\end{aligned}
$$

Thus, $u_{0}$ is a solution for the time-fractional integral equation. It is obvious that $u_{0}$ is a solution for the time-fractional integrodifferential equation $(*)$ whenever $u_{0}$ is a solution for the time-fractional integral equation. This completes the proof.

By considering the proof of last result, one can get that the solution of the time-fractional integrodifferential equation $(*)$ is in the form

$$
\begin{aligned}
u(x, t)= & -\lambda \int_{0}^{t} \frac{(t-\tau)^{\alpha-1}}{\Gamma(\alpha)} f\left(x, \tau, u(x, \tau), \frac{\partial^{q}}{\partial t^{q}} u(x, \tau)\right) d \tau \\
& -\mu \int_{0}^{t} \frac{(t-\tau)^{\alpha+\beta-1}}{\Gamma(\alpha+\beta)} g(x, \tau, u(x, \tau)) d \tau \\
& +\frac{t Q+t^{2} R}{2 N} \lambda \\
& \times \int_{0}^{1} \frac{(1-\tau)^{\alpha-\delta-2}}{\Gamma(\alpha-\delta-1)} f\left(x, \tau, u(x, \tau), \frac{\partial^{q}}{\partial t^{q}} u(x, \tau)\right) d \tau
\end{aligned}
$$

$$
\begin{aligned}
& +\frac{t Q+t^{2} R}{2 N} \mu \\
& \times \int_{0}^{1} \frac{(1-\tau)^{\alpha+\beta-\delta-2}}{\Gamma(\alpha+\beta-\delta-1)} g(x, \tau, u(x, \tau)) d \tau \\
& +\frac{t^{2} S-t M}{2 N} \lambda \\
& \times \int_{0}^{1} \frac{(1-\tau)^{\alpha-\delta-1}}{\Gamma(\alpha-\delta)} f\left(x, \tau, u(x, \tau), \frac{\partial^{q}}{\partial t^{q}} u(x, \tau)\right) d \tau \\
& +\frac{t^{2} S-t M}{2 N} \mu \int_{0}^{1} \frac{(1-\tau)^{\alpha+\beta-\delta-1}}{\Gamma(\alpha+\beta-\delta)} g(x, \tau, u(x, \tau)) d \tau \\
& +\frac{t M-t^{2} S}{2 N} \lambda \\
& \times \int_{0}^{\eta} \frac{(\eta-\tau)^{\alpha-\delta-1}}{\Gamma(\alpha-\delta)} f\left(x, \tau, u(x, \tau), \frac{\partial^{q}}{\partial t^{q}} u(x, \tau)\right) d \tau \\
& +\frac{t M-t^{2} S}{2 N} \mu \int_{0}^{\eta} \frac{(\eta-\tau)^{\alpha+\beta-\delta-1}}{\Gamma(\alpha+\beta-\delta)} g(x, \tau, u(x, \tau)) d \tau,
\end{aligned}
$$

where $Q=2 \Gamma(2-\delta)\left(1-\eta^{2-\delta}\right), R=\left(\eta^{1-\delta}-1\right) \Gamma(3-\delta), S=(1-$ $\delta) \Gamma(3-\delta), M=2 \Gamma(3-\delta)$, and $N=\left[\eta^{1-\delta}(2-\delta)+\eta^{2-\delta}(\delta-1)-1\right]$ with $\eta^{1-\delta}, \eta^{2-\delta} \neq 1$.

Theorem 4. Let $p_{1} \in(0,1), p_{2} \in(0, \alpha-\delta), p=\min \left\{p_{1}, p_{2}\right\}$, $L, m \in L_{1 / p}\left([0,1], \mathbb{R}^{+}\right)$, and $\psi: \mathbb{R}^{+} \rightarrow \mathbb{R}^{+}$be $a$ nondecreasing bounded function. Suppose that $f: J \times X^{2} \rightarrow X$ and $g: J \times X \rightarrow X$ are continuous functions such that $|f(x, t, u, w)-f(x, t, v, z)| \leq L(t)(|u-v|+|w-z|)$ and $\mid g(x, t, u(x, t) \mid \leq m(t) \psi(\|u\|)$ for all $(x, t) \in J$ and $u, v, w, z \in$ X. Put

$$
\begin{aligned}
\gamma:=|\lambda|[ & \frac{1}{\Gamma(\alpha)}\left(\frac{1-p}{\alpha-p}\right)^{1-p}+\frac{1}{\Gamma(\alpha-q)}\left(\frac{1-p}{\alpha-q-p}\right)^{1-p} \\
& +\left|\frac{Q+R}{2 N}\right| \frac{1}{\Gamma(\alpha-\delta-1)}\left(\frac{1-p}{\alpha-\delta-p-1}\right)^{1-p} \\
& +\left|\frac{(2-q) Q+2 R}{2 N \Gamma(3-q)}\right| \frac{1}{\Gamma(\alpha-\delta-1)} \\
& \times\left(\frac{1-p}{\alpha-\delta-p-1}\right)^{1-p} \\
& +\left|\frac{S}{2 N}\right| \frac{\left(1+\eta^{\alpha-\delta-p}\right.}{\Gamma(\alpha-\delta)}\left(\frac{1-p}{\alpha-\delta-p}\right)^{1-p} \\
& +\left|\frac{2 S-(2-q) M}{2 N \Gamma(3-q)}\right| \frac{\left(1+\eta^{\alpha-\delta-p}\right)}{\Gamma(\alpha-\delta)} \\
& \left.\times\left(\frac{1-p}{\alpha-\delta-p}\right)^{1-p}\right]\|L\|_{1 / p}
\end{aligned}
$$


If $\gamma<1$, then the time-fractional integrodifferential equation $(*)$ has at least one solution.

Proof. First, put

$$
\begin{aligned}
w=|\lambda| & +|\mu| b \\
\times[ & \frac{1}{\Gamma(\alpha+\beta)}\left(\frac{1-p}{\alpha+\beta-p}\right)^{1-p}+\frac{1}{\Gamma(\alpha+\beta-q)} \\
& \times\left(\frac{1-p}{\alpha+\beta-q-p}\right)^{1-p}+\left|\frac{Q+R}{2 N}\right| \frac{1}{\Gamma(\alpha+\beta-\delta-1)} \\
& \times\left(\frac{1-p}{\alpha+\beta-\delta-p-1}\right)^{1-p}+\left|\frac{(2-q) Q+2 R}{2 N \Gamma(3-q)}\right| \\
& \times \frac{1}{\Gamma(\alpha+\beta-\delta-1)}\left(\frac{1-p}{\alpha+\beta-\delta-p-1}\right)^{1-p} \\
& +\left|\frac{S}{2 N}\right| \frac{\left(1+\eta^{\alpha+\beta-\delta-p}\right.}{\Gamma(\alpha+\beta-\delta)}\left(\frac{1-p}{\alpha+\beta-\delta-p}\right)^{1-p} \\
& +\left|\frac{2 S-(2-q) M}{2 N \Gamma(3-q)}\right| \frac{\left(1+\eta^{\alpha+\beta-\delta-p}\right)}{\Gamma(\alpha+\beta-\delta)} \\
& \left.\times\left(\frac{1-p}{\alpha+\beta-\delta-p}\right)^{1-p}\right]\|m\|_{1 / p},
\end{aligned}
$$

where

$$
\begin{aligned}
\sigma=K( & \frac{1}{\Gamma(\alpha+1)}+\frac{1}{\Gamma(\alpha-q+1)} \\
& +\left|\frac{Q+R}{2 N}\right| \frac{1}{\Gamma(\alpha-\delta)}+\left|\frac{(2-q) Q+2 R}{2 N \Gamma(3-q)}\right| \frac{1}{\Gamma(\alpha-\delta)} \\
& +\left|\frac{S}{2 N}\right|\left(\frac{1+\eta^{\alpha-\delta}}{\Gamma(\alpha-\delta+1)}\right) \\
& \left.+\left|\frac{2 S-(2-q) M}{2 N \Gamma(3-q)}\right|\left(\frac{1+\eta^{\alpha-\delta}}{\Gamma(\alpha-\delta+1)}\right)\right) .
\end{aligned}
$$

Now, choose $r \geq w /(1-\gamma)$ and consider the closed, convex, and bounded subset $B_{r}=\{u \in X:\|u\| \leq r\}$ of $X$. Define the operator $\Phi: B_{r} \rightarrow X$ by

$$
\begin{aligned}
& (\Phi u)(x, t) \\
& =-\lambda \int_{0}^{t} \frac{(t-\tau)^{\alpha-1}}{\Gamma(\alpha)} f\left(x, \tau, u(x, \tau), \frac{\partial^{q}}{\partial t^{q}} u(x, \tau)\right) d \tau \\
& -\mu \int_{0}^{t} \frac{(t-\tau)^{\alpha+\beta-1}}{\Gamma(\alpha+\beta)} g(x, \tau, u(x, \tau)) d \tau+\frac{t Q+t^{2} R}{2 N} \\
& \quad \times\left[\lambda \int_{0}^{1} \frac{(1-\tau)^{\alpha-\delta-2}}{\Gamma(\alpha-\delta-1)} f\left(x, \tau, u(x, \tau), \frac{\partial^{q}}{\partial t^{q}} u(x, \tau)\right) d \tau\right. \\
& \left.\quad+\mu \int_{0}^{1} \frac{(1-\tau)^{\alpha+\beta-\delta-2}}{\Gamma(\alpha+\beta-\delta-1)} g(x, \tau, u(x, \tau)) d \tau\right]
\end{aligned}
$$

$$
\begin{aligned}
& +\frac{t^{2} S-t M}{2 N} \\
& \times\left[\lambda \int_{0}^{1} \frac{(1-\tau)^{\alpha-\delta-1}}{\Gamma(\alpha-\delta)} f\left(x, \tau, u(x, \tau), \frac{\partial^{q}}{\partial t^{q}} u(x, \tau)\right) d \tau\right. \\
& \quad+\mu \int_{0}^{1} \frac{(1-\tau)^{\alpha+\beta-\delta-1}}{\Gamma(\alpha+\beta-\delta)} g(x, \tau, u(x, \tau)) d \tau \\
& -\lambda \int_{0}^{\eta} \frac{(\eta-\tau)^{\alpha-\delta-1}}{\Gamma(\alpha-\delta)} f\left(x, \tau, u(x, \tau), \frac{\partial^{q}}{\partial t^{q}} u(x, \tau)\right) d \tau \\
& \left.\quad-\mu \int_{0}^{\eta} \frac{(\eta-\tau)^{\alpha+\beta-\delta-1}}{\Gamma(\alpha+\beta-\delta)} g(x, \tau, u(x, \tau)) d \tau\right],
\end{aligned}
$$

for all $(x, t) \in J$. Now, decompose $\Phi$ by $\Phi=\Phi_{1}+\Phi_{2}$, where

$\left(\Phi_{1} u\right)(x, t)$

$$
=-\lambda \int_{0}^{t} \frac{(t-\tau)^{\alpha-1}}{\Gamma(\alpha)} f\left(x, \tau, u(x, \tau), \frac{\partial^{q}}{\partial t^{q}} u(x, \tau)\right) d \tau
$$$$
+\frac{t Q+t^{2} R}{2 N} \lambda
$$$$
\times \int_{0}^{1} \frac{(1-\tau)^{\alpha-\delta-2}}{\Gamma(\alpha-\delta-1)} f\left(x, \tau, u(x, \tau), \frac{\partial^{q}}{\partial t^{q}} u(x, \tau)\right) d \tau
$$$$
+\frac{t^{2} S-t M}{2 N} \lambda
$$$$
\times\left[\int_{0}^{1} \frac{(1-\tau)^{\alpha-\delta-1}}{\Gamma(\alpha-\delta)} f\left(x, \tau, u(x, \tau), \frac{\partial^{q}}{\partial t^{q}} u(x, \tau)\right) d \tau\right.
$$$$
\left.-\int_{0}^{\eta} \frac{(\eta-\tau)^{\alpha-\delta-1}}{\Gamma(\alpha-\delta)} f\left(x, \tau, u(x, \tau), \frac{\partial^{q}}{\partial t^{q}} u(x, \tau)\right) d \tau\right],
$$

$\left(\Phi_{2} u\right)(x, t)$

$$
\begin{array}{r}
=-\mu \int_{0}^{t} \frac{(t-\tau)^{\alpha+\beta-1}}{\Gamma(\alpha+\beta)} g(x, \tau, u(x, \tau)) d \tau \\
+\frac{t Q+t^{2} R}{2 N} \mu \int_{0}^{1} \frac{(1-\tau)^{\alpha+\beta-\delta-2}}{\Gamma(\alpha+\beta-\delta-1)} g(x, \tau, u(x, \tau)) d \tau \\
+\frac{t^{2} S-t M}{2 N}\left[\mu \int_{0}^{1} \frac{(1-\tau)^{\alpha+\beta-\delta-1}}{\Gamma(\alpha+\beta-\delta)} g(x, \tau, u(x, \tau)) d \tau\right. \\
\left.-\mu \int_{0}^{\eta} \frac{(\eta-\tau)^{\alpha+\beta-\delta-1}}{\Gamma(\alpha+\beta-\delta)} g(x, \tau, u(x, \tau)) d \tau\right],
\end{array}
$$


for all $(x, t) \in J$. Now, we show that $\Phi\left(B_{r}\right) \subset B_{r}$. Let $\sup _{x, t \in[0,1]}|f(x, t, 0,0)| \leq K,(x, t) \in J$, and $u \in B_{r}$. Then, we have

$\left|\Phi_{1} u(x, t)\right|$

$$
\begin{aligned}
& \leq|\lambda| \mid \int_{0}^{t} \frac{(t-\tau)^{\alpha-1}}{\Gamma(\alpha)} \\
& \times {\left[f\left(x, \tau, u(x, \tau), \frac{\partial^{q}}{\partial t^{q}} u(x, \tau)\right)\right.} \\
&\quad-f(x, \tau, 0,0)+f(x, \tau, 0,0)] d \tau \mid \\
&+\left|\frac{Q+R}{2 N}\right||\lambda|
\end{aligned}
$$$$
\times \mid \int_{0}^{1} \frac{(1-\tau)^{\alpha-\delta-2}}{\Gamma(\alpha-\delta-1)}\left[f\left(x, \tau, u(x, \tau), \frac{\partial^{q}}{\partial t^{q}} u(x, \tau)\right)\right.
$$$$
-f(x, \tau, 0,0)+f(x, \tau, 0,0)] d \tau \mid
$$$$
+\left|\frac{S}{2 N}\right||\lambda| \mid \int_{0}^{1} \frac{(1-\tau)^{\alpha-\delta-1}}{\Gamma(\alpha-\delta)}
$$$$
\times\left[f\left(x, \tau, u(x, \tau), \frac{\partial^{q}}{\partial t^{q}} u(x, \tau)\right)\right.
$$$$
-f(x, \tau, 0,0)+f(x, \tau, 0,0)] d \tau \mid
$$$$
+\left|\frac{S}{2 N}\right||\lambda| \mid \int_{0}^{\eta} \frac{(\eta-\tau)^{\alpha-\delta-1}}{\Gamma(\alpha-\delta)}
$$$$
\times\left[f\left(x, \tau, u(x, \tau), \frac{\partial^{q}}{\partial t^{q}} u(x, \tau)\right)\right.
$$$$
-f(x, \tau, 0,0)+f(x, \tau, 0,0)] d \tau \mid
$$$$
\leq|\lambda| r\left[\int_{0}^{t} \frac{(t-\tau)^{\alpha-1}}{\Gamma(\alpha)} L(\tau) d \tau\right]+\frac{|\lambda| K}{\Gamma(\alpha+1)}
$$$$
+\left|\frac{Q+R}{2 N}\right||\lambda| r\left[\int_{0}^{1} \frac{(1-\tau)^{\alpha-\delta-2}}{\Gamma(\alpha-\delta-1)} L(\tau) d \tau\right]
$$$$
+\left|\frac{Q+R}{2 N}\right||\lambda| \frac{K}{\Gamma(\alpha-\delta)}
$$$$
+\left|\frac{S}{2 N}\right||\lambda| r\left[\int_{0}^{1} \frac{(1-\tau)^{\alpha-\delta-1}}{\Gamma(\alpha-\delta)} L(\tau) d \tau\right]
$$$$
+\left|\frac{S}{2 N}\right||\lambda| \frac{K}{\Gamma(\alpha-\delta+1)}
$$$$
+\left|\frac{S}{2 N}\right||\lambda| r\left[\int_{0}^{\eta} \frac{(\eta-\tau)^{\alpha-\delta-1}}{\Gamma(\alpha-\delta)} L(\tau) d \tau\right]
$$

$$
\begin{aligned}
& +\left|\frac{S}{2 N}\right||\lambda| \frac{K \eta^{\alpha-\delta}}{\Gamma(\alpha-\delta+1)} \\
& \leq \frac{|\lambda| r}{\Gamma(\alpha)}\left(\int_{0}^{t}(t-\tau)^{(\alpha-1) /(1-p)} d \tau\right)^{1-p}\left(\int_{0}^{1} L^{1 / p}(\tau) d \tau\right)^{p} \\
& +\left|\frac{Q+R}{2 N}\right| \frac{|\lambda| r}{\Gamma(\alpha-\delta-1)}\left(\int_{0}^{1}(1-\tau)^{(\alpha-\delta-2) /(1-p)} d \tau\right)^{1-p} \\
& +\left(\int_{0}^{1} L^{1 / p}(\tau) d \tau\right)^{p} \\
& +\left|\frac{S}{2 N}\right| \frac{|\lambda| r}{\Gamma(\alpha-\delta)}\left(\int_{0}^{1}(1-\tau)^{(\alpha-\delta-1) /(1-p)} d \tau\right)^{1-p} \\
& +\left(\int_{0}^{1} L^{1 / p}(\tau) d \tau\right)^{p} \\
& +\left|\frac{S}{2 N}\right| \frac{|\lambda| r}{\Gamma(\alpha-\delta)}\left(\int_{0}^{\eta}(\eta-\tau)^{(\alpha-\delta-1) /(1-p)} d \tau\right)^{1-p} \\
& +\left(\int_{0}^{1} L^{1 / p}(\tau) d \tau\right)^{p} \\
& +|\lambda| K\left(\frac{1}{\Gamma(\alpha+1)}+\left|\frac{Q+R}{2 N}\right| \frac{1}{\Gamma(\alpha-\delta)}\right. \\
& +|\lambda| r \mid \frac{1}{\Gamma(\alpha)}\left(\frac{1-p}{\alpha-p}\right)^{1-p} \\
& +\left|\frac{Q+R}{2 N}\right| \frac{1}{\Gamma(\alpha-\delta-1)}\left(\frac{1}{\alpha-\delta-p-1}\right)^{1-p} \\
& \left.\left.+\mid \frac{1+\eta^{\alpha-\delta}}{\Gamma(\alpha-\delta+1)}\right)\right)
\end{aligned}
$$

and similarly

$$
\begin{aligned}
\left|\frac{\partial^{q}}{\partial t^{q}} \Phi_{1} u(x, t)\right| & \\
\leq|\lambda| \mid \int_{0}^{t} \frac{(t-\tau)^{\alpha-q-1}}{\Gamma(\alpha-q)}[ & f\left(x, \tau, u(x, \tau), \frac{\partial^{q}}{\partial t^{q}} u(x, \tau)\right) \\
& -f(x, \tau, 0,0)+f(x, \tau, 0,0)] d \tau \mid
\end{aligned}
$$




$$
\begin{aligned}
& +\left|\frac{(2-q) Q+2 R}{2 N \Gamma(3-q)}\right||\lambda| \\
& \times \mid \int_{0}^{1} \frac{(1-\tau)^{\alpha-\delta-2}}{\Gamma(\alpha-\delta-1)}\left[f\left(x, \tau, u(x, \tau), \frac{\partial^{q}}{\partial t^{q}} u(x, \tau)\right)\right. \\
& -f(x, \tau, 0,0)+f(x, \tau, 0,0)] d \tau \mid \\
& +\left|\frac{2 S-(2-q) M}{2 N \Gamma(3-q)}\right||\lambda| \\
& \times \mid \int_{0}^{1} \frac{(1-\tau)^{\alpha-\delta-1}}{\Gamma(\alpha-\delta)}\left[f\left(x, \tau, u(x, \tau), \frac{\partial^{q}}{\partial t^{q}} u(x, \tau)\right)\right. \\
& -f(x, \tau, 0,0)+f(x, \tau, 0,0)] d \tau \\
& +\left|\frac{2 S-(2-q) M}{2 N \Gamma(3-q)}\right||\lambda| \\
& \times \mid \int_{0}^{\eta} \frac{(\eta-\tau)^{\alpha-\delta-1}}{\Gamma(\alpha-\delta)}\left[f\left(x, \tau, u(x, \tau), \frac{\partial^{q}}{\partial t^{q}} u(x, \tau)\right)\right. \\
& -f(x, \tau, 0,0)+f(x, \tau, 0,0)] d \tau \mid \\
& \leq \frac{|\lambda| r}{\Gamma(\alpha-q)}\left(\int_{0}^{t}(t-\tau)^{(\alpha-q-1) /(1-p)} d \tau\right)^{1-p} \\
& \times\left(\int_{0}^{1} L^{1 / p}(\tau) d \tau\right)^{p} \\
& +\left|\frac{(2-q) Q+2 R}{2 N \Gamma(3-q)}\right| \frac{|\lambda| r}{\Gamma(\alpha-\delta-1)} \\
& \times\left(\int_{0}^{1}(1-\tau)^{(\alpha-\delta-2) /(1-p)} d \tau\right)^{1-p}\left(\int_{0}^{1} L^{1 / p}(\tau) d \tau\right)^{p} \\
& +\left|\frac{2 S-(2-q) M}{2 N \Gamma(3-q)}\right| \frac{|\lambda| r}{\Gamma(\alpha-\delta)} \\
& \times\left(\int_{0}^{1}(1-\tau)^{(\alpha-\delta-1) /(1-p)} d \tau\right)^{1-p}\left(\int_{0}^{1} L^{1 / p}(\tau) d \tau\right)^{p} \\
& +\left|\frac{2 S-(2-q) M}{2 N \Gamma(3-q)}\right| \frac{|\lambda| r}{\Gamma(\alpha-\delta)} \\
& \times\left(\int_{0}^{\eta}(\eta-\tau)^{(\alpha-\delta-1) /(1-p)} d \tau\right)^{1-p}\left(\int_{0}^{1} L^{1 / p}(\tau) d \tau\right)^{p} \\
& +|\lambda| K\left(\frac{1}{\Gamma(\alpha-q+1)}+\left|\frac{(2-q) Q+2 R}{2 N \Gamma(3-q)}\right| \frac{1}{\Gamma(\alpha-\delta)}\right. \\
& \left.+\left|\frac{2 S-(2-q) M}{2 N \Gamma(3-q)}\right|\left(\frac{1+\eta^{\alpha-\delta}}{\Gamma(\alpha-\delta+1)}\right)\right)
\end{aligned}
$$$$
\begin{aligned}
\leq|\lambda| r[ & \frac{1}{\Gamma(\alpha-q)}\left(\frac{1-p}{\alpha-q-p}\right)^{1-p}+\left|\frac{(2-q) Q+2 R}{2 N \Gamma(3-q)}\right| \\
& \times \frac{1}{\Gamma(\alpha-\delta-1)}\left(\frac{1-p}{\alpha-\delta-p-1}\right)^{1-p} \\
& +\left|\frac{2 S-(2-q) M}{2 N \Gamma(3-q)}\right| \frac{1}{\Gamma(\alpha-\delta)}\left(\frac{1-p}{\alpha-\delta-p}\right)^{1-p} \\
& \left.+\left|\frac{2 S-(2-q) M}{2 N \Gamma(3-q)}\right| \frac{\eta^{\alpha-\delta-p}}{\Gamma(\alpha-\delta)}\left(\frac{1-p}{\alpha-\delta-p}\right)^{1-p}\right]
\end{aligned}
$$$$
\times\|L\|_{1 / p}
$$$$
+|\lambda| K\left(\frac{1}{\Gamma(\alpha-q+1)}+\left|\frac{(2-q) Q+2 R}{2 N \Gamma(3-q)}\right| \frac{1}{\Gamma(\alpha-\delta)}\right.
$$$$
\left.+\left|\frac{2 S-(2-q) M}{2 N \Gamma(3-q)}\right|\left(\frac{1+\eta^{\alpha-\delta}}{\Gamma(\alpha-\delta+1)}\right)\right) \text {. }
$$

Thus, we get

$\left\|\Phi_{1} u(x, t)\right\|$

$$
\begin{aligned}
& \leq|\lambda| r[ \frac{1}{\Gamma(\alpha)}\left(\frac{1-p}{\alpha-p}\right)^{1-p}+\frac{1}{\Gamma(\alpha-q)}\left(\frac{1-p}{\alpha-q-p}\right)^{1-p} \\
&+\left|\frac{Q+R}{2 N}\right| \frac{1}{\Gamma(\alpha-\delta-1)}\left(\frac{1-p}{\alpha-\delta-p-1}\right)^{1-p} \\
&+\left|\frac{(2-q) Q+2 R}{2 N \Gamma(3-q)}\right| \frac{1}{\Gamma(\alpha-\delta-1)} \\
& \times\left(\frac{1-p}{\alpha-\delta-p-1}\right)^{1-p} \\
&+\mid \frac{S}{2 N \mid} \frac{\left(1+\eta^{\alpha-\delta-p}\right)}{\Gamma(\alpha-\delta)}\left(\frac{1-p}{\alpha-\delta-p}\right)^{1-p} \\
&+\left|\frac{2 S-(2-q) M}{2 N \Gamma(3-q)}\right| \frac{\left(1+\eta^{\alpha-\delta-p}\right)}{\Gamma(\alpha-\delta)} \\
&\left.\times\left(\frac{1-p}{\alpha-\delta-p}\right)^{1-p}\right]\|L\|_{1 / p} \\
&+|\lambda| K\left(\frac{1}{\Gamma(\alpha+1)}+\frac{1}{\Gamma(\alpha-q+1)}+\frac{Q+R}{2 N} \mid \frac{1}{\Gamma(\alpha-\delta)}\right. \\
& \quad+\left|\frac{S}{2 N}\right|\left(\frac{1+\eta^{\alpha-\delta}}{\Gamma(\alpha-\delta+1)}\right)
\end{aligned}
$$


8

Mathematical Problems in Engineering

If $\sup _{u \in X} \psi(\|u\|) \leq b$, then we have

$$
\left|\Phi_{2} u(x, t)\right|
$$

$$
\leq|\mu|\left|\int_{0}^{t} \frac{(t-\tau)^{\alpha+\beta-1}}{\Gamma(\alpha+\beta)} g(x, \tau, u(x, \tau)) d \tau\right|
$$

$+\left|\frac{Q+R}{2 N}\right||\mu|\left|\int_{0}^{1} \frac{(1-\tau)^{\alpha+\beta-\delta-2}}{\Gamma(\alpha+\beta-\delta-1)} g(x, \tau, u(x, \tau)) d \tau\right|$

$+\left|\frac{S}{2 N}\right||\mu|\left|\int_{0}^{1} \frac{(1-\tau)^{\alpha+\beta-\delta-1}}{\Gamma(\alpha+\beta-\delta)} g(x, \tau, u(x, \tau)) d \tau\right|$

$+\left|\frac{S}{2 N}\right||\mu|\left|\int_{0}^{\eta} \frac{(\eta-\tau)^{\alpha+\beta-\delta-1}}{\Gamma(\alpha+\beta-\delta)} g(x, \tau, u(x, \tau)) d \tau\right|$

$\begin{aligned} \leq & \frac{|\mu| b}{\Gamma(\alpha+\beta)}\left(\int_{0}^{t}(t-\tau)^{(\alpha+\beta-1) /(1-p)} d \tau\right)^{1-p} \\ & \times\left(\int_{0}^{1} m^{1 / p}(\tau) d \tau\right)^{p}+\left|\frac{Q+R}{2 N}\right| \frac{|\mu| b}{\Gamma(\alpha+\beta-\delta-1)}\end{aligned}$

$\times\left(\int_{0}^{1}(1-\tau)^{(\alpha+\beta-\delta-2) /(1-p)} d \tau\right)^{1-p}$

$\times\left(\int_{0}^{1} m^{1 / p}(\tau) d \tau\right)^{p}$

$+\left|\frac{S}{2 N}\right| \frac{|\mu| b}{\Gamma(\alpha+\beta-\delta)}\left(\int_{0}^{1}(1-\tau)^{(\alpha+\beta-\delta-1) /(1-p)}\right)^{1-p}$

$\times\left(\int_{0}^{1} m^{1 / p}(\tau) d \tau\right)^{p}$

$+\left|\frac{S}{2 N}\right| \frac{|\mu| b}{\Gamma(\alpha+\beta-\delta)}\left(\int_{0}^{\eta}(\eta-\tau)^{(\alpha+\beta-\delta-1) /(1-p)}\right)^{1-p}$

$\times\left(\int_{0}^{1} m^{1 / p}(\tau) d \tau\right)^{p}$

$\leq|\mu| b\left[\frac{1}{\Gamma(\alpha+\beta)}\left(\frac{1-p}{\alpha+\beta-p}\right)^{1-p}+\left|\frac{Q+R}{2 N}\right|\right.$

$\times \frac{1}{\Gamma(\alpha+\beta-\delta-1)}\left(\frac{1-p}{\alpha+\beta-\delta-p-1}\right)^{1-p}$

$+\left|\frac{S}{2 N}\right| \frac{1}{\Gamma(\alpha+\beta-\delta)}\left(\frac{1-p}{\alpha+\beta-\delta-p}\right)^{1-p}$

$$
\left.+\left|\frac{S}{2 N}\right| \frac{\eta^{\alpha+\beta-\delta-p}}{\Gamma(\alpha+\beta-\delta)}\left(\frac{1-p}{\alpha+\beta-\delta-p}\right)^{1-p}\right\rfloor
$$

$\times\|m\|_{1 / p}$

and similarly

$$
\begin{aligned}
& \left|\frac{\partial^{q}}{\partial t^{q}} \Phi_{2} u(x, t)\right| \\
& \leq|\mu|\left|\int_{0}^{t} \frac{(t-\tau)^{\alpha+\beta-q-1}}{\Gamma(\alpha+\beta-q)} g(x, \tau, u(x, \tau)) d \tau\right| \\
& +\left|\frac{(2-q) Q+2 R}{2 N \Gamma(3-q)}\right||\mu| \\
& \times\left|\int_{0}^{1} \frac{(1-\tau)^{\alpha+\beta-\delta-2}}{\Gamma(\alpha+\beta-\delta-1)} g(x, \tau, u(x, \tau)) d \tau\right| \\
& +\left|\frac{2 S-(2-q) M}{2 N \Gamma(3-q)}\right||\mu| \\
& \times\left|\int_{0}^{1} \frac{(1-\tau)^{\alpha+\beta-\delta-1}}{\Gamma(\alpha+\beta-\delta)} g(x, \tau, u(x, \tau)) d \tau\right| \\
& +\left|\frac{2 S-(2-q) M}{2 N \Gamma(3-q)}\right||\mu| \\
& \times\left|\int_{0}^{\eta} \frac{(\eta-\tau)^{\alpha+\beta-\delta-1}}{\Gamma(\alpha+\beta-\delta)} g(x, \tau, u(x, \tau)) d \tau\right| \\
& \leq \frac{|\mu| b}{\Gamma(\alpha+\beta-q)}\left(\int_{0}^{t}(t-\tau)^{(\alpha+\beta-q-1) /(1-p)} d \tau\right)^{1-p} \\
& \times\left(\int_{0}^{1} m^{1 / p}(\tau) d \tau\right)^{p} \\
& +\left|\frac{(2-q) Q+2 R}{2 N \Gamma(3-q)}\right| \frac{|\mu| b}{\Gamma(\alpha+\beta-\delta-1)} \\
& \times\left(\int_{0}^{1}(1-\tau)^{(\alpha+\beta-\delta-2) /(1-p)} d \tau\right)^{1-p} \\
& \times\left(\int_{0}^{1} m^{1 / p}(\tau) d \tau\right)^{p}+\left|\frac{2 S-(2-q) M}{2 N \Gamma(3-q)}\right| \\
& \times \frac{|\mu| b}{\Gamma(\alpha+\beta-\delta)}\left(\int_{0}^{1}(1-\tau)^{(\alpha+\beta-\delta-1) /(1-p)} d \tau\right)^{1-p} \\
& \times\left(\int_{0}^{1} m^{1 / p}(\tau) d \tau\right)^{p}+\left|\frac{2 S-(2-q) M}{2 N \Gamma(3-q)}\right| \\
& \times \frac{|\mu| b}{\Gamma(\alpha+\beta-\delta)}\left(\int_{0}^{\eta}(\eta-\tau)^{(\alpha+\beta-\delta-1) /(1-p)} d \tau\right)^{1-p} \\
& \times\left(\int_{0}^{1} m^{1 / p}(\tau) d \tau\right)^{p} \\
& \leq|\mu| b\left[\frac{1}{\Gamma(\alpha+\beta-q)}\left(\frac{1-p}{\alpha+\beta-q-p}\right)^{1-p}\right. \\
& +\left|\frac{(2-q) Q+2 R}{2 N \Gamma(3-q)}\right|
\end{aligned}
$$




$$
\begin{aligned}
& \times \frac{1}{\Gamma(\alpha+\beta-\delta-1)}\left(\frac{1-p}{\alpha+\beta-\delta-p-1}\right)^{1-p} \\
& +\left|\frac{2 S-(2-q) M}{2 N \Gamma(3-q)}\right| \frac{1}{\Gamma(\alpha+\beta-\delta)} \\
& \times\left(\frac{1-p}{\alpha+\beta-\delta-p}\right)^{1-p}+\left|\frac{2 S-(2-q) M}{2 N \Gamma(3-q)}\right| \\
& \left.\times \frac{\eta^{\alpha+\beta-\delta-p}}{\Gamma(\alpha+\beta-\delta)}\left(\frac{1-p}{\alpha+\beta-\delta-p}\right)^{1-p}\right]\|m\|_{1 / p} .
\end{aligned}
$$

Hence,

$$
\begin{aligned}
\left\|\Phi_{2} u(x, t)\right\| & \\
\leq|\mu| b[ & \frac{1}{\Gamma(\alpha+\beta)}\left(\frac{1-p}{\alpha+\beta-p}\right)^{1-p}+\frac{1}{\Gamma(\alpha+\beta-q)} \\
& \times\left(\frac{1-p}{\alpha+\beta-q-p}\right)^{1-p}+\left|\frac{Q+R}{2 N}\right| \\
& \times \frac{1}{\Gamma(\alpha+\beta-\delta-1)}\left(\frac{1-p}{\alpha+\beta-\delta-p-1}\right)^{1-p} \\
& +\left|\frac{(2-q) Q+2 R}{2 N \Gamma(3-q)}\right| \frac{1}{\Gamma(\alpha+\beta-\delta-1)} \\
& \times\left(\frac{1-p}{\alpha+\beta-\delta-p-1}\right)^{1-p}+\left|\frac{S}{2 N}\right| \\
& \times \frac{\left(1+\eta^{\alpha+\beta-\delta-p}\right)}{\Gamma(\alpha+\beta-\delta)}\left(\frac{1-p}{\alpha+\beta-\delta-p}\right)^{1-p} \\
& +\left|\frac{2 S-(2-q) M}{2 N \Gamma(3-q)}\right| \frac{\left(1+\eta^{\alpha+\beta-\delta-p}\right)}{\Gamma(\alpha+\beta-\delta)} \\
& \left.\times\left(\frac{1-p}{\alpha+\beta-\delta-p}\right)^{1-p}\right]\|m\|_{1 / p}
\end{aligned}
$$

and so

$\|\Phi u(x, t)\|$

$$
\begin{aligned}
& \leq\left\|\Phi_{1} u(x, t)\right\|+\left\|\Phi_{2} u(x, t)\right\| \\
& \leq|\lambda| r\left[\frac{1}{\Gamma(\alpha)}\left(\frac{1-p}{\alpha-p}\right)^{1-p}+\frac{1}{\Gamma(\alpha-q)}\left(\frac{1-p}{\alpha-q-p}\right)^{1-p}\right. \\
& +\left|\frac{Q+R}{2 N}\right| \frac{1}{\Gamma(\alpha-\delta-1)}\left(\frac{1-p}{\alpha-\delta-p-1}\right)^{1-p} \\
& +\left|\frac{(2-q) Q+2 R}{2 N \Gamma(3-q)}\right| \frac{1}{\Gamma(\alpha-\delta-1)}
\end{aligned}
$$

$$
\begin{aligned}
& \times\left(\frac{1-p}{\alpha-\delta-p-1}\right)^{1-p}+\left|\frac{S}{2 N}\right| \frac{\left(1+\eta^{\alpha-\delta-p}\right)}{\Gamma(\alpha-\delta)} \\
& \times\left(\frac{1-p}{\alpha-\delta-p}\right)^{1-p}+\left|\frac{2 S-(2-q) M}{2 N \Gamma(3-q)}\right| \\
& \left.\times \frac{\left(1+\eta^{\alpha-\delta-p}\right)}{\Gamma(\alpha-\delta)}\left(\frac{1-p}{\alpha-\delta-p}\right)^{1-p}\right]\|L\|_{1 / p} \\
& +|\lambda| K\left(\frac{1}{\Gamma(\alpha+1)}+\frac{1}{\Gamma(\alpha-q+1)}+\left|\frac{Q+R}{2 N}\right|\right. \\
& \times \frac{1}{\Gamma(\alpha-\delta)}+\left|\frac{(2-q) Q+2 R}{2 N \Gamma(3-q)}\right| \frac{1}{\Gamma(\alpha-\delta)} \\
& +\left|\frac{S}{2 N}\right|\left(\frac{1+\eta^{\alpha-\delta}}{\Gamma(\alpha-\delta+1)}\right) \\
& \left.+\left|\frac{2 S-(2-q) M}{2 N \Gamma(3-q)}\right|\left(\frac{1+\eta^{\alpha-\delta}}{\Gamma(\alpha-\delta+1)}\right)\right) \\
& +|\mu| b\left[\frac{1}{\Gamma(\alpha+\beta)}\left(\frac{1-p}{\alpha+\beta-p}\right)^{1-p}+\frac{1}{\Gamma(\alpha+\beta-q)}\right. \\
& \times\left(\frac{1-p}{\alpha+\beta-q-p}\right)^{1-p}+\left|\frac{Q+R}{2 N}\right| \\
& \times \frac{1}{\Gamma(\alpha+\beta-\delta-1)}\left(\frac{1-p}{\alpha+\beta-\delta-p-1}\right)^{1-p} \\
& +\left|\frac{(2-q) Q+2 R}{2 N \Gamma(3-q)}\right| \frac{1}{\Gamma(\alpha+\beta-\delta-1)} \\
& \times\left(\frac{1-p}{\alpha+\beta-\delta-p-1}\right)^{1-p}+\left|\frac{S}{2 N}\right| \\
& \times \frac{\left(1+\eta^{\alpha+\beta-\delta-p}\right)}{\Gamma(\alpha+\beta-\delta)}\left(\frac{1-p}{\alpha+\beta-\delta-p}\right)^{1-p} \\
& +\left|\frac{2 S-(2-q) M}{2 N \Gamma(3-q)}\right| \frac{\left(1+\eta^{\alpha+\beta-\delta-p}\right)}{\Gamma(\alpha+\beta-\delta)} \\
& \left.\times\left(\frac{1-p}{\alpha+\beta-\delta-p}\right)^{1-p}\right]\|m\|_{1 / p}
\end{aligned}
$$

$\leq \gamma r+w \leq r$.

This implies that $\Phi B_{r} \subset B_{r}$. Now, we show that $\Phi_{1}$ is continuous. Let $x, t \in[0,1]$ and $\left\{u_{n}\right\}$ be a sequence with $u_{n} \rightarrow u$. Then, we have

$$
\begin{aligned}
& \left\|\Phi_{1} u_{n}(x, t)-\Phi_{1} u(x, t)\right\| \\
& \leq\left[\frac{|\lambda|\|L\|_{1 / p}}{\Gamma(\alpha)}\left(\frac{1-p}{\alpha-p}\right)^{1-p}+\frac{|\lambda|\|L\|_{1 / p}}{\Gamma(\alpha-q)}\left(\frac{1-p}{\alpha-q-p}\right)^{1-p}\right. \\
& \quad+\left|\frac{Q+R}{2 N}\right| \frac{|\lambda|\|L\|_{1 / p}}{\Gamma(\alpha-\delta-1)}\left(\frac{1-p}{\alpha-\delta-p-1}\right)^{1-p}
\end{aligned}
$$




$$
\begin{aligned}
& +\left|\frac{(2-q) Q+2 R}{2 N \Gamma(3-q)}\right| \frac{|\lambda|\|L\|_{1 / p}}{\Gamma(\alpha-\delta-1)}\left(\frac{1-p}{\alpha-\delta-p-1}\right)^{1-p} \\
& +\left|\frac{S}{2 N}\right| \frac{|\lambda|\|L\|_{1 / p}\left(1+\eta^{\alpha-\delta-p}\right)}{\Gamma(\alpha-\delta)}\left(\frac{1-p}{\alpha-\delta-p}\right)^{1-p} \\
& +\left|\frac{2 S-(2-q) M}{2 N \Gamma(3-q)}\right| \frac{|\lambda|\|L\|_{1 / p}\left(1+\eta^{\alpha-\delta-p}\right)}{\Gamma(\alpha-\delta)} \\
& \left.\times\left(\frac{1-p}{\alpha-\delta-p}\right)^{1-p}\right]\left\|u_{n}-u\right\| .
\end{aligned}
$$

Thus, $\Phi_{1}$ is continuous. On the other hand, $\Phi_{1}$ is a $\gamma$ contraction because

$$
\begin{aligned}
\left\|\Phi_{1} u(x, t)-\Phi_{1} v(x, t)\right\| \\
\leq \mid \frac{|\lambda|\|L\|_{1 / p}}{\Gamma(\alpha)}\left(\frac{1-p}{\alpha-p}\right)^{1-p}+\frac{|\lambda|\|L\|_{1 / p}}{\Gamma(\alpha-q)}\left(\frac{1-p}{\alpha-q-p}\right)^{1-p} \\
+\left|\frac{Q+R}{2 N}\right| \frac{|\lambda|\|L\|_{1 / p}}{\Gamma(\alpha-\delta-1)}\left(\frac{1-p}{\alpha-\delta-p-1}\right)^{1-p} \\
+\left|\frac{(2-q) Q+2 R}{2 N \Gamma(3-q)}\right| \frac{|\lambda|\|L\|_{1 / p}}{\Gamma(\alpha-\delta-1)}\left(\frac{1-p}{\alpha-\delta-p-1}\right)^{1-p} \\
+\left|\frac{S}{2 N \mid}\right| \frac{|\lambda|\|L\|_{1 / p}\left(1+\eta^{\alpha-\delta-p}\right)}{\Gamma(\alpha-\delta)}\left(\frac{1-p}{\alpha-\delta-p}\right)^{1-p} \\
+\left|\frac{2 S-(2-q) M}{2 N \Gamma(3-q)}\right| \frac{|\lambda|\|L\|_{1 / p}\left(1+\eta^{\alpha-\delta-p}\right)}{\Gamma(\alpha-\delta)} \\
\left.\quad \times\left(\frac{1-p}{\alpha-\delta-p}\right)^{1-p}\right]\|u-v\|,
\end{aligned}
$$

for all $u, v \in B_{r}$. Now, we show that $\Phi_{2}$ is a compact map. We showed that $\Phi_{2}$ is uniformly bounded. Now, we show that $\Phi_{2}$ maps the bounded sets into equicontinuous sets. Let $\left(x, t_{1}\right),\left(x, t_{2}\right) \in J$ such that $t_{1}<t_{2}$. Then, we have

$$
\begin{aligned}
& \left|\Phi_{2} u\left(x, t_{2}\right)-\Phi_{2} u\left(x, t_{1}\right)\right| \\
& \leq \frac{|\mu|}{\Gamma(\alpha+\beta)} \int_{0}^{t_{1}}\left[\left(t_{2}-\tau\right)^{\alpha+\beta-1}-\left(t_{1}-\tau\right)^{\alpha+\beta-1}\right] \\
& \quad \times|g(x, \tau, u(x, \tau))| d \tau \\
& \quad+\frac{|\mu|}{\Gamma(\alpha+\beta)} \int_{t_{1}}^{t_{2}}\left(t_{2}-\tau\right)^{\alpha+\beta-1}|g(x, \tau, u(x, \tau))| d \tau \\
& \quad+\frac{\left|t_{2}-t_{1}\right||Q|+\left|t_{2}^{2}-t_{1}^{2}\right||R|}{|2 N|}|\mu| \\
& \quad \times \int_{0}^{1} \frac{(1-\tau)^{\alpha+\beta-\delta-2}}{\Gamma(\alpha+\beta-\delta-1)}|g(x, \tau, u(x, \tau))| d \tau
\end{aligned}
$$

$$
\begin{aligned}
& +\frac{\left|t_{2}^{2}-t_{1}^{2}\right||S|-\left|t_{2}-t_{1}\right||M|}{|2 N|}|\mu| \\
& \times\left[\int_{0}^{1} \frac{(1-\tau)^{\alpha+\beta-\delta-1}}{\Gamma(\alpha+\beta-\delta)}|g(x, \tau, u(x, \tau))| d \tau\right. \\
& \left.-\int_{0}^{\eta} \frac{(\eta-\tau)^{\alpha+\beta-\delta-1}}{\Gamma(\alpha+\beta-\delta)}|g(x, \tau, u(x, \tau))| d \tau\right] \\
& \leq \frac{|\mu| b}{\Gamma(\alpha+\beta)} \int_{0}^{t_{1}}\left[\left(t_{2}-\tau\right)^{\alpha+\beta-1}-\left(t_{1}-\tau\right)^{\alpha+\beta-1}\right] m(\tau) d \tau \\
& +\frac{|\mu| b}{\Gamma(\alpha+\beta)} \int_{t_{1}}^{t_{2}}\left(t_{2}-\tau\right)^{\alpha+\beta-1} m(\tau) d \tau \\
& +\left(\frac{\left|t_{2}-t_{1}\right||Q|+\left|t_{2}^{2}-t_{1}^{2}\right||R|}{|2 N|}\right)|\mu| b \\
& \times \int_{0}^{1} \frac{(1-\tau)^{\alpha+\beta-\delta-2}}{\Gamma(\alpha+\beta-\delta-1)} m(\tau) d \tau \\
& +\left(\frac{\left|t_{2}^{2}-t_{1}^{2}\right||S|-\left|t_{2}-t_{1}\right||M|}{|2 N|}\right)|\mu| b \\
& \times\left[\int_{0}^{1} \frac{(1-\tau)^{\alpha+\beta-\delta-1}}{\Gamma(\alpha+\beta-\delta)} m(\tau) d \tau\right. \\
& \left.-\int_{0}^{\eta} \frac{(\eta-\tau)^{\alpha+\beta-\delta-1}}{\Gamma(\alpha+\beta-\delta)} m(\tau) d \tau\right], \\
& \left|\frac{\partial^{q}}{\partial t^{q}} \Phi_{2} u\left(x, t_{2}\right)-\frac{\partial^{q}}{\partial t^{q}} \Phi_{2} u\left(x, t_{1}\right)\right| \\
& \leq \frac{|\mu| b}{\Gamma(\alpha+\beta-q)} \\
& \times \int_{0}^{t_{1}}\left[\left(t_{2}-\tau\right)^{\alpha+\beta-q-1}-\left(t_{1}-\tau\right)^{\alpha+\beta-q-1}\right] m(\tau) d \tau \\
& +\frac{|\mu| b}{\Gamma(\alpha+\beta-q)} \int_{t_{1}}^{t_{2}}\left(t_{2}-\tau\right)^{\alpha+\beta-q-1} m(\tau) d \tau \\
& +\left(\left(\frac{\left|t_{2}^{1-q}-t_{1}^{1-q}\right|}{\Gamma(2-q)}|Q|\right.\right. \\
& \left.\left.+\frac{\left|t_{2}^{2-q}-t_{1}^{2-q}\right|}{\Gamma(3-q)}|R|\right) \times|2 N|^{-1}\right)|\mu| b \\
& \times \int_{0}^{1} \frac{(1-\tau)^{\alpha+\beta-\delta-2}}{\Gamma(\alpha+\beta-\delta-1)} m(\tau) d \tau \\
& +\left(\left(\frac{2\left|t_{2}^{2-q}-t_{1}^{2-q}\right|}{\Gamma(3-q)}|S|\right.\right. \\
& \left.\left.-\frac{\left|t_{2}^{1-q}-t_{1}^{1-q}\right|}{\Gamma(2-q)}|M|\right) \times|2 N|^{-1}\right)|\mu| b
\end{aligned}
$$




$$
\begin{aligned}
& \times\left[\int_{0}^{1} \frac{(1-\tau)^{\alpha+\beta-\delta-1}}{\Gamma(\alpha+\beta-\delta)} m(\tau) d \tau\right. \\
& \left.\quad-\int_{0}^{\eta} \frac{(\eta-\tau)^{\alpha+\beta-\delta-1}}{\Gamma(\alpha+\beta-\delta)} m(\tau) d \tau\right],
\end{aligned}
$$

for all $u \in B_{r}$. Hence, $\left\|\Phi_{2} u\left(x, t_{2}\right)-\Phi_{2} u\left(x, t_{1}\right)\right\| \rightarrow 0$ as $t_{2} \rightarrow t_{1}$. By using the Arzela-Ascoli theorem, we get that the operator $\Phi_{2}$ is completely continuous and so $\Phi_{2}$ is compact on $J$. Now by using Lemma 1 , the operator $\Phi=\Phi_{1}+\Phi_{2}$ is a condensing map on $B_{r}$ and so $\Phi$ has a fixed point by using Theorem 2. Now by using Lemma 3, it is easy to see that the fixed point of $\Phi$ is a solution for the time-fractional integrodifferential equation $(*)$.

Example 5. Let $\alpha=5 / 2, \beta=3 / 4, q=3 / 2, \delta=5 / 4, \eta=2 / 3$, $\lambda=1 / 1000, \mu=1$, and $p=3 / 4$. Now, consider the timefractional integrodifferential equation

$$
\begin{aligned}
-\frac{\partial^{5 / 2}}{\partial t^{5 / 2}} u(x, t)= & \frac{1}{1000}\left(\frac{t|u|}{1+|u|}+\frac{t\left|\left(\partial^{3 / 2} / \partial t^{3 / 2}\right) u\right|}{1+\left|\left(\partial^{3 / 2} / \partial t^{3 / 2}\right) u\right|}\right) \\
& +I_{* t}^{3 / 4}\left(\frac{t^{2}|u|^{3}}{1+|u|^{3}}\right)
\end{aligned}
$$

via the boundary conditions $u(x, 0)=0,\left(\partial^{9 / 4} / \partial t^{9 / 4}\right)$ $u(x, 0)=\left(\partial^{9 / 4} / \partial t^{9 / 4}\right) u(x, 1)$, and $\left(\partial^{5 / 4} / \partial t^{5 / 4}\right) u(x, 1)-$ $\left(\partial^{5 / 4} / \partial t^{5 / 4}\right) u(x, 2 / 3)=0$. Define the maps $f: J \times X^{2} \rightarrow$ $X$ by $f\left(x, t, u(x, t),\left(\partial^{3 / 2} / \partial t^{3 / 2}\right) u(x, t)\right)=t|u| /(1+|u|)+$ $t\left|\left(\partial^{3 / 2} / \partial t^{3 / 2}\right) u\right| /\left(1+\left|\left(\partial^{3 / 2} / \partial t^{3 / 2}\right) u\right|\right)$ and $g: J \times X \rightarrow X$ by $g(x, t, u(x, t))=t^{2}|u|^{3} /\left(1+|u|^{3}\right)$. Define $L(t)=t$ and $m(t)=t^{2}$ for all $t$. Then, we have $\|L\|_{1 / p}=\|t\|_{4 / 3}=0.077$. Define $\psi: \mathbb{R}^{+} \rightarrow \mathbb{R}^{+}$by $\psi(z)=1$ for all $z$. It is easy to check that

$$
\begin{aligned}
& \mid f\left(x, t, u(x, t), \frac{\partial^{3 / 2}}{\partial t^{3 / 2}} u(x, t)\right) \\
& \quad-f\left(x, t, v(x, t), \frac{\partial^{3 / 2}}{\partial t^{3 / 2}} v(x, t)\right) \mid \\
& \quad \leq L(t)\left(|u-v|+\left|\frac{\partial^{3 / 2}}{\partial t^{3 / 2}} u-\frac{\partial^{3 / 2}}{\partial t^{3 / 2}} v\right|\right)
\end{aligned}
$$

and $|g(x, t, u(x, t))| \leq m(t) \psi(\|u\|)$ for all $u, v \in X$ and $t$. One can calculate that $\|m\|_{1 / p}=\left\|t^{2}\right\|_{4 / 3}=(3 / 11)^{3 / 4}$ and $\gamma \simeq 0.002579$. Now by using Theorem 4 , the time-fractional integrodifferential equation has a solution.

\section{Conclusion}

A time-fractional integrodifferential equation via three-point boundary value conditions was investigated and the existence of the solution was proved for three-point boundary value conditions. One example was studied in detail. For particular forms of the functions $f$ and $g$ and of the investigated equation and for various values of $2 \leq \alpha<3,0<\beta<$ $1,1 \leq \delta<2,0 \leq \eta \leq 1, \lambda$, and $\mu$ we can reobtain the forms of several nonlinear time-fractional differential equations describing the complex phenomena which arise in science and engineering.

\section{Conflict of Interests}

The authors declare that there is no conflict of interests regarding the publication of this paper.

\section{Acknowledgment}

Research of the authors was supported by Azarbaijan Shahid Madani University.

\section{References}

[1] I. Podlubny, Fractional Differential Equations, Academic Press, San Diego, Calif, USA, 1999.

[2] S. G. Samko, A. A. Kilbas, and O. I. Marichev, Fractional Integrals and Derivatives: Theory and Applications, Gordon and Breach Science Publishers, Yverdon, Switzerland, 1993.

[3] R. Hilfer, Applications of Fractional Calculus in Physics, Academic Press, New York, NY, USA, 1999.

[4] W. Wyss, "The fractional Black-Scholes equation," Fractional Calculus and Applied Analysis, vol. 3, no. 1, pp. 51-61, 2000.

[5] J. Sabatier, O. P. Agrawal, and J. A. Tenreiro Machado, Advances in Fractional Calculus: Theoretical Developments and Applications in Physics and Engineering, Springer, 2007.

[6] G. Maione, "On the Laguerre rational approximation to fractional discrete derivative and integral operators," IEEE Transactions on Automatic Control, vol. 58, no. 6, pp. 1579-1585, 2013.

[7] H. Zhang, F. Liu, P. Zhuang, I. Turner, and V. Anh, "Numerical analysis of a new space-time variable fractional order advection-dispersion equation," Applied Mathematics and Computation, vol. 242, pp. 541-550, 2014.

[8] R. P. Agarwal, S. K. Ntouyas, B. Ahmad, and M. S. Alhothuali, "Existence of solutions for integro-differential equations of fractional order with nonlocal three-point fractional boundary conditions," Advances in Difference Equations, vol. 2013, article 128, 2013.

[9] B. Ahmad, S. K. Ntouyas, and A. Alsaedi, "On fractional differential inclusions with anti-periodic type integral boundary conditions," Boundary Value Problems, vol. 2013, article 82, 2013.

[10] A. Alsaedi, S. K. Ntouyas, and B. Ahmad, "Existence results for Langevin fractional differential inclusions involving two fractional orders with four-point multiterm fractional integral boundary conditions," Abstract and Applied Analysis, vol. 2013, Article ID 869837, 17 pages, 2013.

[11] Z. Bai and W. Sun, "Existence and multiplicity of positive solutions for singular fractional boundary value problems," Computers \& Mathematics with Applications, vol. 63, no. 9, pp. 1369-1381, 2012.

[12] D. Baleanu, R. P. Agarwal, H. Mohammadi, and S. Rezapour, "Some existence results for a nonlinear fractional differential equation on partially ordered Banach spaces," Boundary Value Problems, vol. 2013, article 112, 2013. 
[13] D. Baleanu, H. Mohammadi, and S. Rezapour, "The existence of solutions for a nonlinear mixed problem of singular fractional differential equations," Advances in Difference Equations, vol. 2013, article 359, 2013.

[14] D. Baleanu, H. Mohammadi, and S. Rezapour, "Positive solutions of an initial value problem for nonlinear fractional differential equations," Abstract and Applied Analysis, vol. 2012, Article ID 837437, 7 pages, 2012.

[15] D. Baleanu, S. Rezapour, and H. Mohammadi, "Some existence results on nonlinear fractional differential equations," Philosophical Transactions of the Royal Society of London, vol. 371, Article ID 20120144, 2013.

[16] X. Liu and Z. Liu, "Existence results for fractional differential inclusions with multivalued term depending on lower-order derivative," Abstract and Applied Analysis, vol. 2012, Article ID 423796, 24 pages, 2012.

[17] P. D. Phung and L. X. Truong, "On a fractional differential inclusion with integral boundary conditions in Banach space," Fractional Calculus and Applied Analysis, vol. 16, no. 3, pp. 538$558,2013$.

[18] L. Debnath and D. D. Bhatta, "Solutions to few linear fractional inhomogeneous partial differential equations in fluid mechanics," Fractional Calculus and Applied Analysis, vol. 7, no. 1, pp. 21-36, 2004.

[19] F. Huang and F. Liu, "The fundamental solution of the space-time fractional advection-dispersion equation," Journal of Applied Mathematics and Computing, vol. 18, no. 1-2, pp. 339350, 2005.

[20] K. S. Miller and B. Ross, An Introduction to the Fractional Calculus and Fractional Differential Equations, John Wiley \& Sons, New York, NY, USA, 1993.

[21] A. Granas and J. Dugundji, Fixed Point Theory, Springer, New York, NY, USA, 2003.

[22] B. N. Sadovskii, “A fixed-point principle," Functional Analysis and Its Applications, vol. 1, no. 2, pp. 151-153, 1967.

[23] E. Zeidler, Nonlinear Functional Analysis and its Application: Fixed Point-Theorems, Springer, 1986. 


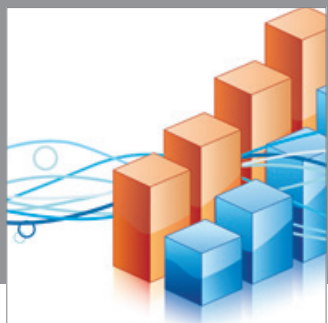

Advances in

Operations Research

mansans

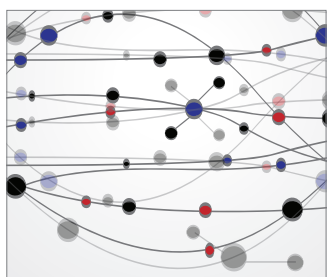

The Scientific World Journal
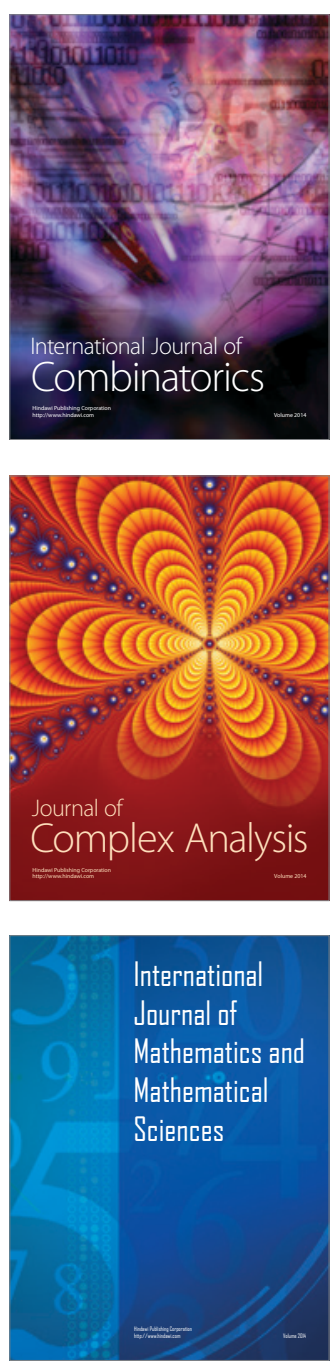
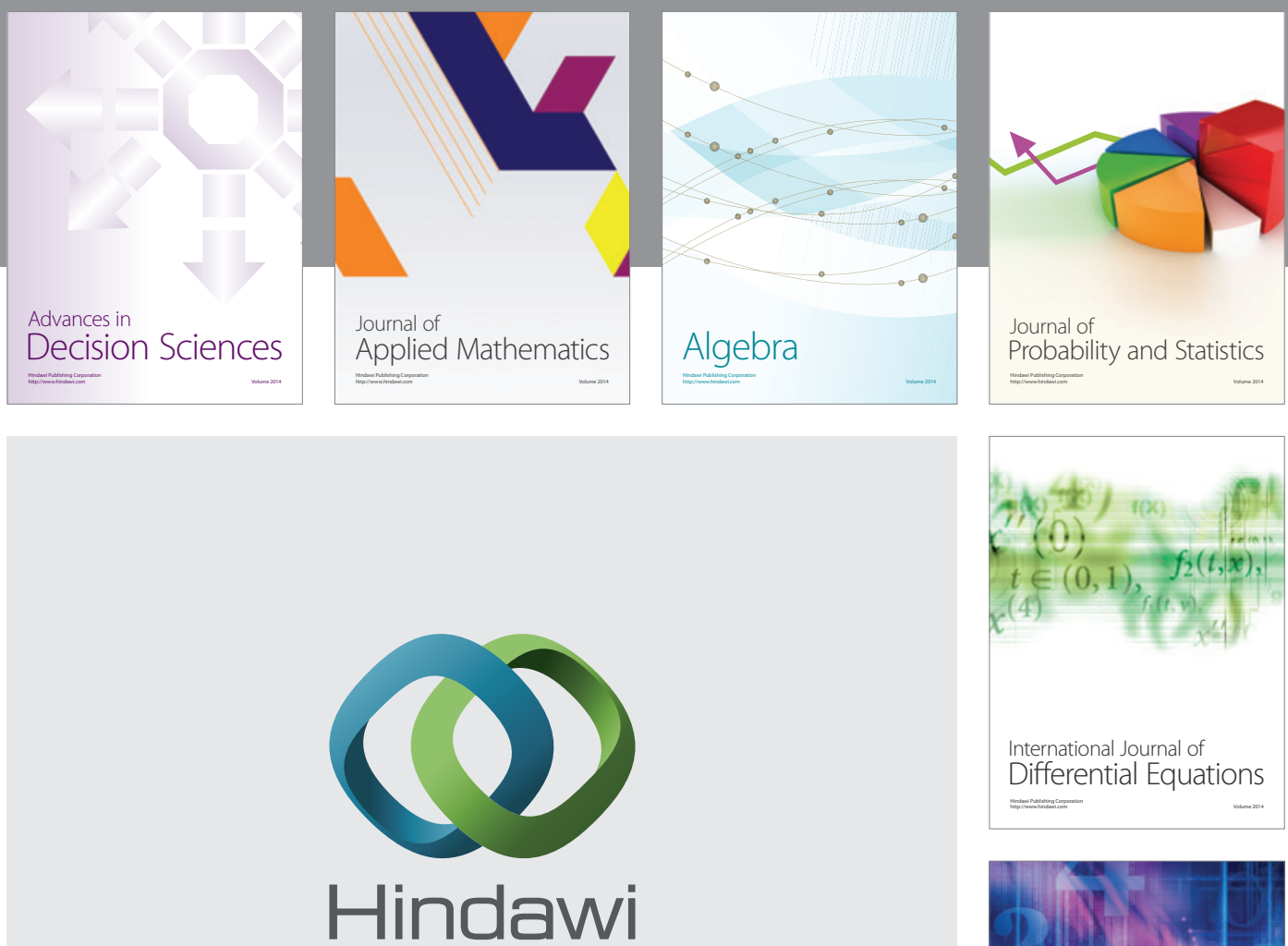

Submit your manuscripts at http://www.hindawi.com
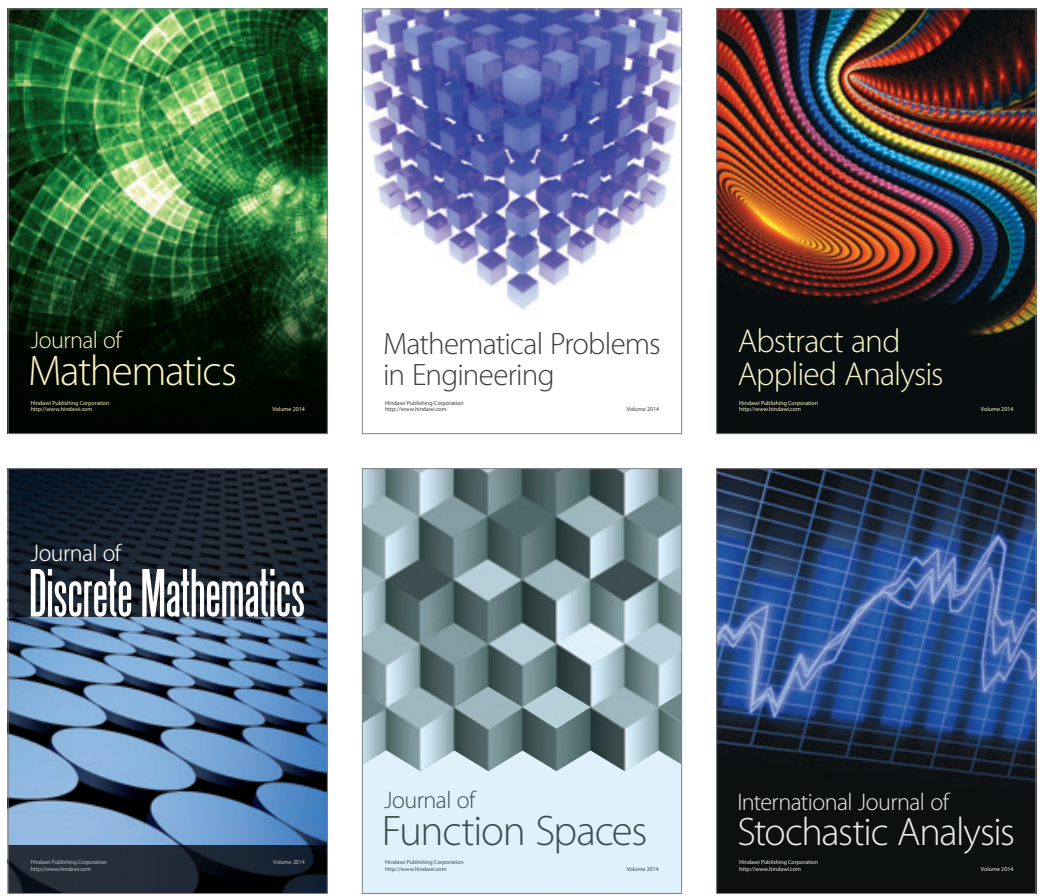

Journal of

Function Spaces

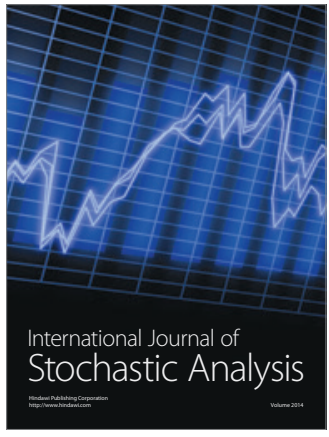

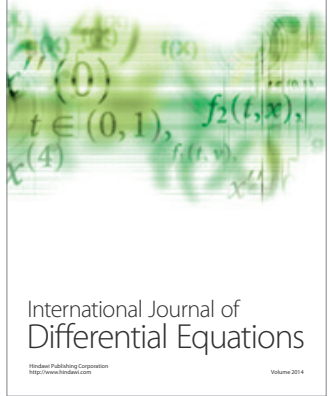
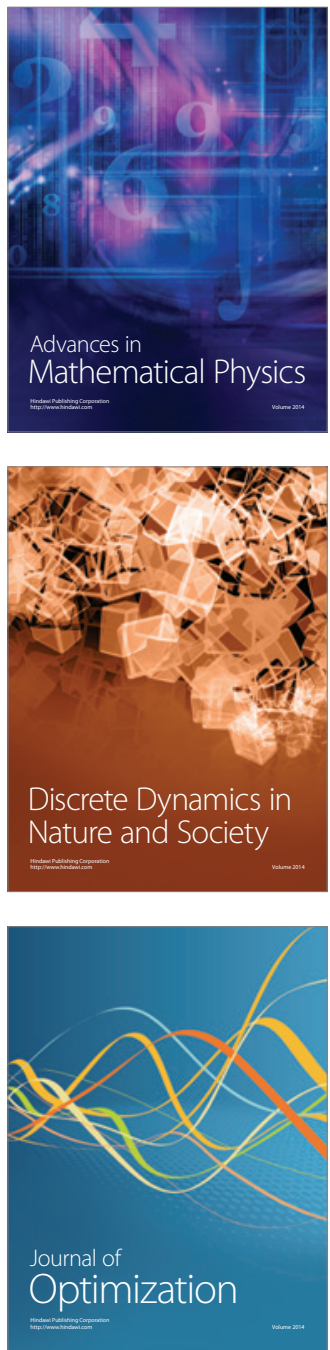